\title{
The Diversity of Food Consumption in Romania
}

\author{
Cecilia ALEXANDRI ${ }^{1}$, Cristian KEVORCHIAN ${ }^{2)}$ \\ 1) Institute of Agricultural Economics, Bucharest, Romania; cecilia@eadr.ro \\ 2) Institute of Agricultural Economics, Bucharest, Romania; \\ ${ }^{*}$ Corresponding author, e-mail: cristian.kevorkian@gmail.com
}

BulletinUASVM Horticulture 72(1) / 2015

Print ISSN 1843-5254, Electronic ISSN 1843-5394

DOI:10.15835/buasvmcn-hort:10747

\begin{abstract}
The relation between the quantities of food consumed from different products and the socio-economic characteristics of households is investigated in many empirical studies. At the same time, there are few concerns with regard to other aspects related to the consumption behaviour, such as the number of individual food items consumed over a certain period, in other words, dietary variety or diversity. Identification of economic and sociodemographic factors influencing food consumption diversity on the Romanian households is the aim of this paper. Among the investigated factors, we had in view the incomes in the first place, then the household residence area, the educational level as well as other demographic factors. Count measure and Berry index are used to measure food diversity. The data used are those from the Household Budget Survey for the year 2011, $1^{\text {st }}$ quarter. As conclusions, the study reveals a positive correlation between diversity and household incomes. At the same time, food diversity is higher in the case of urban households compared to the rural households. Among the significant demographic factors, it can be mentioned the household head's educational level and the household members' age.
\end{abstract}

Keywords: Berry index, food and nutritional security, food diversity, household level.

\section{INTRODUCTION}

The modelling of dependency between socioeconomic characteristics of households, such as age, household members' incomes, household size, composition and household head's educational level and foodstuffs consumed represents a rich topic in household food security research. Closely linked with the mentioned topic, the modelling of consumption behaviour - such as number and type of foodstuffs consumed in a certain period - reveals important aspects related to dietary variety or diversity. Food consumption diversity is important from several points of view. In the first instance, a mostly diversified diet represents a modality by which people can protect themselves against certain diseases. In this respect, the nutritionists' opinion is that an optimum diet should be composed of quite a large number of different products (Randall, E., et al, 1985). In order to measure diet's diversity, the US Department of Agriculture developed the Healthy Eating Index
(HEI). This index contains 12 components, among which 9 components measure diet adequacy and 3 components refer to moderation in people's food consumption (Guenther P.M., et al, 2013). Another index for dietary diversity assessment, used by FAO, is Household Dietary Diversity Score (HDDS), which is calculated by counting the type of consumed foodstuffs selected from 12 main food groups: 1.Cereals, 2 . Roots and tubers, 3 . Vegetables, 4. Fruit, 5. Meat and offal, 6. Eggs, 7. Fish and seafood, 8. Pulses, beans, nuts, 9. Milk and dairy products, 10 . Oils and fats, 11 . Sugar and honey, 12. Miscellaneous. (Swindale, A., Bilinski, P., 2006).

Knowing how dietary diversity evolves in relation to certain factors is also important for the marketing studies, for adapting the strategies to consumers' needs. At the same time, increasing the food consumption diversity in certain geographic areas has important implications upon the world and regional agri-food trade and upon 
the economic development in general (Lancaster, K., 1990).

The economic literature use the theory of indifference curves (concave curves) for the investigation of consumer preferences, which shows that various combinations of two goods provides consumer equal utility or satisfaction (Dominick, 2008). This approach starts from the assumption that all individuals always eat all the types of available products and cannot explain why certain consumers buy a larger number of products than others, hence being inadequate for the investigation of food diversity demand.

There are certain approaches that link dietary diversity to the level of incomes (Jackson, L.F., 1984). Thus, in the situation when the income level is low, only a part (subset) of available foodstuffs is bought. This pattern is known as the hierarchical demand system. As the consumption level increases, the number of products that go to the consumer basket also increases.

Other studies containing comparisons across countries also reveal the positive correlation between diversity (measured by Herfinfahl index or by entropy index) and the level of incomes, measured by the real incomes per capita (Theil, H., et al., 1983). At the same time, dietary diversity on the household can be positively correlated with certain demographic factors such as the number of members by genders, age groups, and the household members' educational level.

Another important element that has a positive influence upon the dietary diversity, mainly in the rural area, is the size of subsistence economy. Thus, a positive influence upon food diversity at the level of rural households is brought by the participation to irrigation schemes, education, income, gender, access to kitchen garden and presence of a few animals in the rural households (A. Taruvinga, 2013).

\section{MATERIALS AND METHODS}

The simplest method for diversity assessment is to count the number of food products consumed by a household or individual. However, this method does not provide information on the distribution of food quantities consumed on individual basis or on the distribution of expenditures.
The diversity concept has applications in biology, social sciences, physics and economy, where the concentration index is used and represents the reverse of the diversity index (Lee and Brown, 1989). The concentration index used in economics has direct connection with the market, where it is considered that the lower the concentration level, the highest the competition between firms.

An indicator used for measuring the concentration is Herfindhal index, which in fact represents an inverse measure of diversity. Berry Index is a more adequate indicator, defined by deducting the Herfindhal index value from unit:

$$
\mathbf{B}_{i}=1-\sum_{j=1}^{N} s^{2} \boldsymbol{j}
$$

where $\mathrm{BI}_{\mathrm{i}}$ is the Berry Index for household $\mathrm{i}$, and $s_{i, j}$ represents the share of expenditures for product $j$ in the food budget on household $i$. In this paper, we evaluated food diversity for the urban and rural households by using the Berry Index. At the computation of the index has been used the microdata from the Household Budget Surveys from the $1^{\text {st }}$ quarter of the year 2011, the maximum number of possible food products, included into survey, to be consumed on each household is $\mathrm{N}_{\max }=104$. The number of households included in the survey sample database was 7844 .

The Berry Index takes values in the interval $[0,1]$. The fact that $\mathrm{BI}=0$ means that the household bought only one food product in the investigated period, while if $\mathrm{BI}=1^{1}$ this means that the household equally bought all the 104 food products considered. Therefore, for certain categories of households in our country, if the Berry Index has values close to 1 (ie, higher than 0.95 ), means that their diet is very diverse. Meanwhile, if for other households, the index values are low (ie, below 0.70 ), it is considered that the diversity of their food consumption is very low.

\section{RESULTS AND DISCUSSION}

In order to identify the main socio-economic and demographic factors that can influence the dietary diversity of households in Romania the Berry Index values computation was calculated

\footnotetext{
${ }^{1}$ The higher value of $\mathrm{BI}$ is $1-\left(\mathrm{N}_{\max }\right)^{-1}$, where for $\mathrm{N}_{\max }=104, \mathrm{BI}=0.990385$ and round $\mathrm{BI}$ to 1.
} 
for each household. To calculate the index has been taken into account weights of each quantity of purchased food in total food purchases. This operation has been followed by statistical grouping, according to the disposable income, residence areas, educational level of household head and the number of children. An overview of the variation of this index in Romania and Germany it's presented in tab. 1.

The research methodology was mapped on the main directions provided by the specialty literature that investigated the dietary diversity

Tab. 1. Descriptive statistics for Berry Index

\begin{tabular}{lcc}
\hline & Romania & Germany \\
\hline Number of households & 7844 & 4632 \\
\hline Mean & 0.887 & 0.901 \\
\hline Standard Deviation & 0.079 & 0.073 \\
\hline Min & 0.235 & 0.018 \\
\hline Max & 0.971 & 0.977
\end{tabular}

Source: Thiele, S et al., 2003 (for Germany) and own calculations (for Romania) issue (Thiele, S., et al., 2003). These reveal that diversity is influenced by the household socioeconomic characteristics, such as income in the first place, secondly by the size and composition of household, mainly the number of children aged 7 - 17 years, the residence area and size of locality where the household is located and the region. A series of characteristics related to the household head are also important, such as age, educational level and occupation, if the household head has a full-time job. But income remains the most important and direct determinant of diversity. At the same time, the farmer households are characterized by a low diversity of the purchased products. Low income at household level make as an important part of foodstuffs to be produced in their own household, and the consumption of bought dietary food to be at a lower level, as was already mentioned. This aspect is noticeable in Romania, where the rural households have a much lower dietary diversity compared to the urban households (Fig. 1).

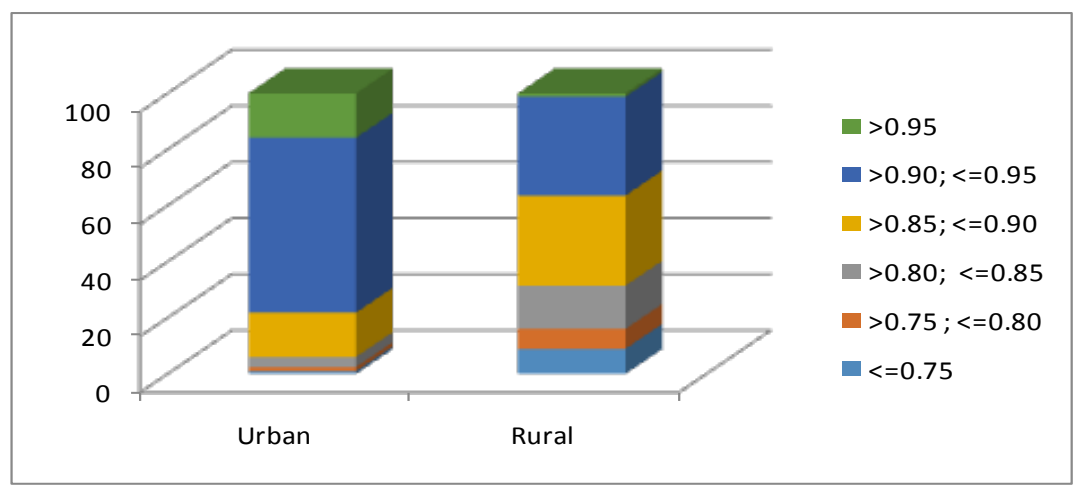

Fig. 1. Distribution of urban and rural households by Berry Index values.

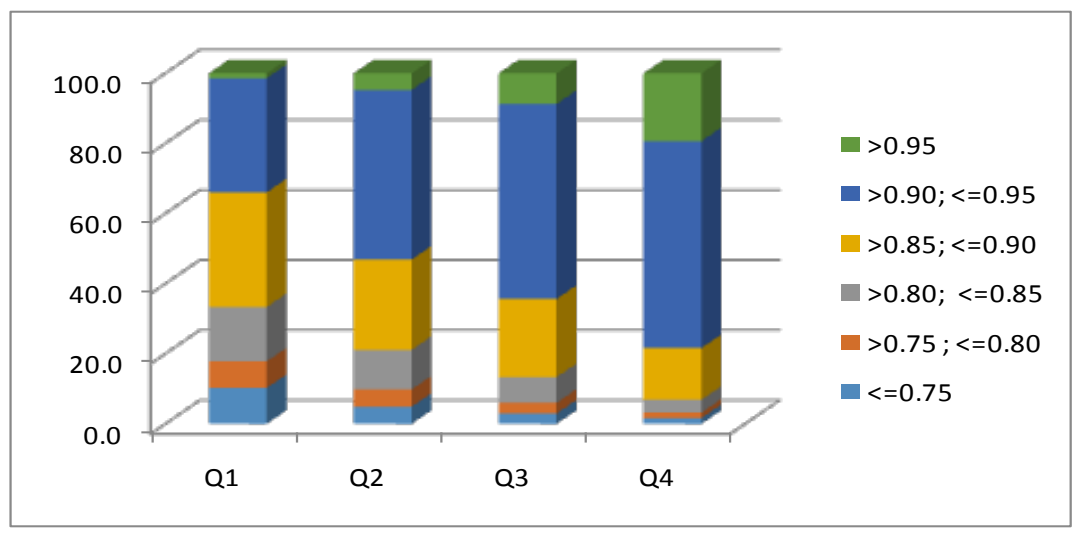

Fig. 2. Distribution of households by Index Berry and income quartiles (\%). 
It is important to mention that the households that have a high dietary diversity, measured with Berry index, for which the index values are higher than 0.90 , account for $68 \%$ of the urban households and only $36 \%$ of the rural households. The rural households have a moderate dietary diversity, most of them $(47 \%)$ being in the interval $(0.80$, 0.90 ) for the Berry Index. The higher food diversity in the case of urban households in comparison with rural households represents an indirect effect of income disparities between households from urban and rural areas. Thus, in 2011, according to the Budget Household Survey (NIS, 2012), urban households had total and cash income higher with $23 \%$, and respectively by $73 \%$ compared to rural households. In addition to the income factor, other factors that influence consumer behaviour and diversity of dietary could be considered including some demographic factors that were already mentioned. In addition, the access to food retail systems and to the transport infrastructure may also influence the behaviour of the consumers from different residence areas.

In this context, our results relative to households' food diet diversity in relation to disposable income fully confirm the economic theory, i.e. a direct link between income growth and diversity of diets. Figure 2 shows that the households from first income quintile is characterized by a medium to low dietary diversity with only $34 \%$ of these to have a high diversity, for which the Berry Index is higher than 0.90. In fourth income quintile, more than $78 \%$ of households have a high dietary diversity, and 20\% have a very high diversity, for which the Berry Index is higher than 0.95. At the same time, the number of households that fall into the high food diversity category increases with incomes, which indicates a consistent statistical relation between income and dietary diversity.

Similar results regarding the influence of income and residence on household food diet diversity were obtained in other European countries (Cupak, A, et al, 2014).

Figure 3 presents the distribution of households' food diet diversity in relation to the household head's educational level. The results are consistent with the economic theory in this approach as well, in the sense that the share of households whose food diversity is high increases with the household head's educational level
(Thiele, S. et al., 2003). This is due, on one hand, to the fact that a higher educational level implies a higher income of the household head, and on the other hand because the educational level contributes to people's becoming aware of the food diversity role for people's health, mainly in the case of children, elderly people and persons with vulnerabilities. Anyhow, it is remarkable that more than $80 \%$ of the households on which the household head graduated a university have a high diversified and a very high diversified diet.

In Figure 4 is emphasized the relation between the number of children on the household and the dietary diversity. The highest share of households with a high diversified diet can be noticed in the category of households with one child, where $57 \%$ of households have calculated Berry Index values higher than 0.90 . As far as the number of children increases, the households fall into zones with medium dietary diversity. Thus, $74 \%$ of the households with 2 children and $84 \%$ of the households with more than 3 children have a diversity index ranging in the interval $(0.85,0.90)$. The relation between the dietary diversity and the number of children does not seem to be very clear, as a household with children should provide them a mostly diversified food from the nutritional point of view; at the same time, in Romania, the households with many children generally have lower household income most often being included in the less-favoured category of the society.

\section{CONCLUSION}

To sum up, it is important to mention that the food consumption diversity of households is an important characteristic of the diet which has a positive influence to nutrition. The level of different nutrients varies with the different foodstuffs. The investigation of the food diversity in different socio-economic and demographic categories of the population is important for the identification of vulnerable categories and for the nutrition programs. The food diversity, which reflect the diet equilibrium, didn't has a standard measure general accepted by administrative and research organisations. Statistical approach of this topic, synthesized in Berry Index, is a solution to study food diversity in many socio-economic contexts and the time point behaviour of some consumer categories. 


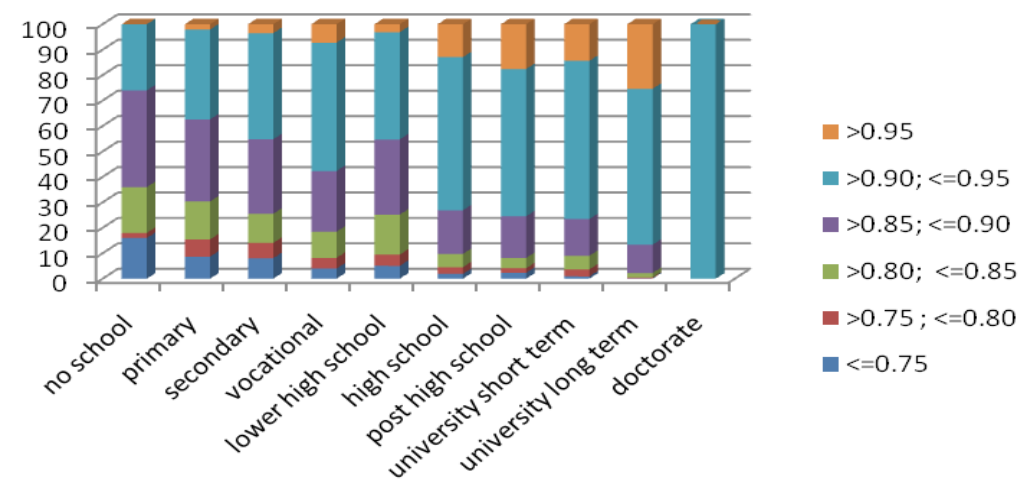

Fig. 3. Distribution of households by Berry Index and by the household head's educational level (\%).

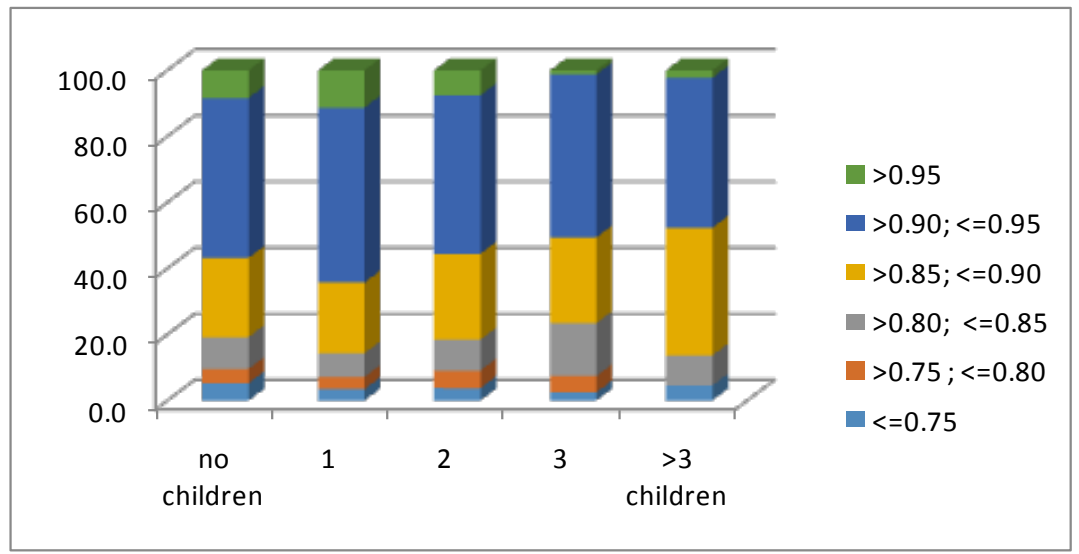

Fig. 4. Distribution of households by Berry Index and by the number of children (\%).

At the same time, the investigation of the variety of consumption provides important elements for the food marketing. Low income, which has a negative influence to food diversity demand induce a negative influence in food commodity markets.

The residence area is also important for the household diet, as the rural households have a less diversified food diet, due to lower incomes and to the high share of self-produced food and subsistence economy. The population's diet in the rural area follows the cycle of seasons, the out of season consumption of fruit and vegetables being very low, for instance.

The number of children is a variable that negatively impacts food diversity, as the households with many children have a lower dietary diversity because most often the incomes on these households are quite low. As a matter of fact, the numerous households with many children in Romania are the most vulnerable from the nutritional point of view, this fact being recognized by the international organizations (FAO, 2012).

Acknowledgments. The research leading to these results has received funding from the European Union's Seventh Framework Program under Grant Agreement n ${ }^{\circ} 290693$ (FoodSecure) and from UEFISCDI (Romania) under contract 198/EU/2012.

\section{REFERENCES}

1. Cupak A, Pokrivcak J, Rizov M (2014). Demand for the Food Diversity in Central and Eastern European Countries: an Evidences from Slovakia, Paper prepared for presentation for the 142th EAAE Seminar, Budapest, 2014.

2. Dominick S (2008). Microeconomics: Theory and Applications .

3. Guenther PM, Casavale KO, Reedy ., Kirkpatrick SI, Hiza HA, Kuczynski KJ, Kahle LL, Krebs-Smith SM (2013). Healthy Eating Index , Academy of Nutrition and Dietetics.

4. Jackson LF (1984). Hierarhic Demand and the Enghel Curve for Variety, Review of Economics and Statistics 66. 
5. Jonq-Ying L, Mark G B (1989). Consumer demand for food diversity, Southern Journal of Agricultural Economics, December 6.

6. Lancaster K (1990). The Economics of Product Variety: a Survey, Marketing Science 9: 189-206.

7. Randall E, Nichaman MZ, Constant CF (1985). Diet diversity and nutrient intake, Journal of American Dietetic Association 85: 830-836.

8. Swindale A, Bilinsky P (2006). Household Dietary Diversity Score (HDDS) for Measurement of Household Food Access: Indicator Guide (v.2), Washington, D.C.: Food and Nutrition Technical Assistance Project, Academy for Educational Development.

9. Taruvinga A, Muchenje V, Muchenje A (2013) Determinants of rural household dietary diversity: The case of Amatole and Nyandeni districts, South Africa, International Journal of Development and Sustainability, Number 4

10. Theil H, Finke R (1983). The consumer's demand for diversity, European Economic Review 23: S395-S400.

11. Thiele S, Weiss C (2003). Consumer demand for food diversity: evidence for

12. Germany, Food Policy 28: 99-115, www.elsevier.com/ locate/foodpol

13. *** FAO (2012), The State of Food and Agriculture.

14. *** NIS (2012), Co-ordinates of living standard in Romania, Population income and consumption in the year 2011. 